\title{
Características de la disfagia en pacientes de un centro de gastroenterología en Bogotá D. C., Colombia
}

\author{
Characteristics of dysphagia in patients of a gastroenterology center in \\ Bogotá D.C., Colombia
}

Juan Sebastián Frías Ordóñez, MD, ${ }^{*}$ Julián David Martínez Marín, MD. ${ }^{2}$

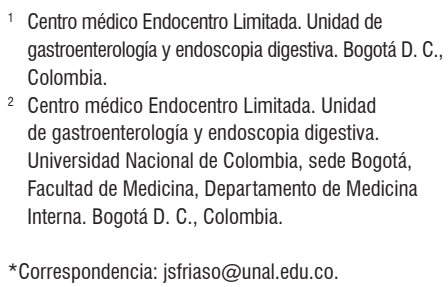

2 Centro médico Endocentro Limitada. Unidad de gastroenterología y endoscopia digestiva. Universidad Nacional de Colombia, sede Bogotá, Facultad de Medicina, Departamento de Medicina Interna. Bogotá D. C., Colombia.

*Correspondencia: jsfriaso@unal.edu.co.

(a)

\begin{abstract}
Resumen
Introducción: la epidemiología de la disfagia está pobremente definida. Es un síntoma que se asocia con el envejecimiento, y en poblaciones jóvenes sigue siendo tema de incertidumbre. También es un problema que no siempre informan los pacientes y es poco comprendido en la población general. Objetivo: describir las características clínicas y epidemiológicas de la disfagia a partir de una muestra de pacientes adultos remitidos a un centro especializado en gastroenterología. Metodología: estudio observacional, descriptivo y retrospectivo, realizado en un centro especializado en gastroenterología en Bogotá D. C. durante 2016, en el cual se revisaron 3148 historias clínicas y se recopilaron aquellas de pacientes cuyo motivo de consulta fuera disfagia o la refirieran como síntoma asociado, y cuyo diagnóstico se confirmara con pruebas complementarias. Resultados: de 3148 historias clínicas, 85 (2,7 \%) presentaron disfagia como motivo de consulta principal o síntoma asociado. La mayoría correspondió a mujeres $(70,5 \%)$. El grupo de edad más afectado fue entre 70 y 79 años. La enfermedad por reflujo gastroesofágico (ERGE) representó el diagnóstico más frecuente. Conclusión: la prevalencia de disfagia encontrada es similar a la reportada en la literatura. La ERGE es causa frecuente de disfagia. La disfagia continúa siendo un problema predominantemente de adultos mayores.
\end{abstract}

\section{Palabras clave}

Trastornos de deglución, prevalencia, epidemiología, endoscopia, adulto.

\section{Abstract}

Introduction: The epidemiology of dysphagia is poorly defined. It is a symptom that is associated with aging but is still a matter of uncertainty in young populations. Also, it is is not always reported by patients and is poorly understood in the general population. Objective: The objective of this study was to describe the clinical and epidemiological characteristics of dysphagia in a sample of adult patients referred to a center specializing in Gastroenterology. Methodology: This is an observational, descriptive and retrospective study carried out in a specialized gastroenterology center in Bogotá DC in 2016. We reviewed 3,148 clinical histories of patients to identify those whose reason for consultation was dysphagia or who were referred to the clinic with dysphagia as an associated symptom and whose diagnoses had been confirmed by complementary tests. Results: Of the 3,148 medical records, $85(2.7 \%)$ presented dysphagia as the main reason for consultation or as an associated symptom. The majority were women $(70.5 \%)$. The largest proportion of those affected were between 70 and 79 years old. Gastroesophageal reflux disease was the most frequent diagnosis. Conclusion: The prevalence of dysphagia found is similar to that reported in the literature. Gastroesophageal reflux disease is a frequent cause of dysphagia. Dysphagia continues to be a problem especially among the elderly.

\section{Keywords (DeCS)}

Swallowing disorders, prevalence, epidemiology, adult, endoscopy. 


\section{INTRODUCCIÓN}

La disfagia es la dificultad que puede presentarse en las fases iniciales de la deglución, o la sensación de obstrucción en el pasaje de alimentos y/o líquidos desde la boca al estómago (1). Según su origen, puede clasificarse en orofaríngea o en esofágica $(2,3)$. La disfagia orofaríngea afecta la boca, la faringe o el esófago proximal, casi siempre se asocia con desórdenes neurológicos (eventos cerebrovasculares, enfermedad de Parkinson o esclerosis lateral amiotrófica), también se puede relacionar con alteraciones anatómicas como el divertículo de Zenker o barras cricofaríngeas (2). La disfagia esofágica afecta al cuerpo esofágico y la unión esofagogástrica, y puede deberse a trastornos de la motilidad esofágica (trastorno motor esofágico primario, esclerodermia), inflamación mucosa (esofagitis eosinofílica, esofagitis por radiación) o anormalidades estructurales (tumores, estenosis péptica, anillos esofágicos o compresión extrínseca) (3).

La epidemiología de la disfagia es pobremente definida a nivel nacional. Por una parte, la prevalencia de la mayoría de enfermedades que pueden provocar disfagia tiende a diferir entre las diferentes regiones y continentes; por otra parte, las tasas de prevalencia varían dependiendo del instrumento o método con el cual se diagnostica la disfagia, y las patologías asociadas con disfagia en grupos de personas jóvenes difieren de las que se presentan en adultos mayores $(3,4)$. Algunos estudios que la han evaluado previamente han incluido otros grupos etarios, pero sus conclusiones son siempre las mismas: la disfagia aparece en edades mayores. En estudios que la han evaluado, se han demostrado resultados variables, estimando una prevalencia comprendida entre $8 \%$ a $22 \%$ en pacientes mayores de 50 años (4-7). Algunos otros estudios realizados exclusivamente con pacientes de hogares geriátricos han llegado a considerar la prevalencia de disfagia en alrededor del $60 \%(8,9)$. La epidemiología en poblaciones más jóvenes sigue siendo tema de incertidumbre (1).

En comparación con los adultos mayores, en pacientes más jóvenes se ha apreciado de modo creciente mayor percepción del problema, y su asociación con enfermedades de distinto espectro como reflujo gastroesofágico o reflujo laringoesofágico (10). La disfagia representa un problema que en gran medida no es notificada y a su vez es pobremente comprendida por la población general, lo que conduce a subdiagnóstico. Por tanto, se hace necesario llegar a una aproximación sobre la frecuencia o la etiología de la disfagia en la población adulta en general en Colombia. En el presente estudio descriptivo se definen las características clínicas y epidemiológicas de la disfagia a partir de una muestra de pacientes adultos remitidos a un centro especializado en gastroenterología.

\section{MATERIALES Y MÉTODOS}

Estudio observacional, descriptivo y retrospectivo en el Centro Médico Endocentro Limitada, en Bogotá D. C., centro especializado en servicios de gastroenterología.

Se revisaron 3148 historias clínicas y se recopilaron aquellas de pacientes cuyo motivo de consulta fuera disfagia o refirieran esta como síntoma asociado, que asistieron a valoración por consulta externa durante el período comprendido entre enero 2 a diciembre 31 de 2016. Se incluyeron pacientes mayores de 16 años. Los pacientes debían tener un diagnóstico confirmado de la disfagia por pruebas diagnósticas complementarias (endoscopia de vías digestivas altas [EVDA], pruebas funcionales o estudios radiográficos).

Los principales datos recopilados fueron la edad, tipo de alimentos con los que se manifestaba la disfagia al momento de la valoración, las pruebas complementarias utilizadas para confirmar su diagnóstico, las comorbilidades asociadas y el diagnóstico relacionado de los pacientes según los hallazgos clínicos, endoscópicos y paraclínicos; y se registraron en una base de datos del programa Microsoft Office Excel ${ }^{\circ}$ versión 2010 para su análisis posterior.

Se garantizó la confidencialidad de la información y ningún registro contenía información sensible sobre la identidad de los pacientes. Se solicitó consentimiento informado; el estudio fue aprobado por el comité de investigación de la institución.

\section{RESULTADOS}

De un total de 3148 historias clínicas revisadas, en 85 (2,7\%) se identificó disfagia como motivo de consulta principal o síntoma asociado. 60 pacientes fueron mujeres $(70,5 \%)$. La edad media fue de 60,6 años (rango de 19 a 92 años). La distribución según sexo y edad se ilustra en la Tabla 1.

De acuerdo con la presencia de signos sugestivos de aspiración o penetración, de los 85 pacientes, $67,05 \%$ presentó disfagia con alimentos sólidos, $3,5 \%$ con líquidos y 29,41 $\%$ con ambas consistencias. Las comorbilidades asociadas encontradas fueron las siguientes: 7 pacientes $(8,24 \%)$ presentaban secuelas de enfermedades cerebrovasculares $(\mathrm{ECV}) \mathrm{u}$ otra enfermedad neurológica, 6 pacientes $(7,05$ $\%)$ con enfermedades reumatológicas que comprometían la motilidad gastrointestinal y $5(5,88 \%)$ con antecedentes de cirugías del tracto gastrointestinal alto. Mientras que 27 pacientes $(31,76 \%)$ no presentaban comorbilidades definidas al momento de la consulta. En la Figura 1 se ilustran las principales comorbilidades encontradas. 
Tabla 1. Distribución de pacientes que presentaron disfagia como motivo de consulta principal o síntoma asociado según sexo y edad

\begin{tabular}{|c|c|c|}
\hline & $\begin{array}{c}\text { Número de } \\
\text { pacientes }(n=85)\end{array}$ & $\%$ \\
\hline \multicolumn{3}{|l|}{ Género } \\
\hline Masculino & 25 & 29,41 \\
\hline Femenino & 60 & 70,58 \\
\hline \multicolumn{3}{|l|}{ Edad } \\
\hline $15-19$ & 1 & 1,17 \\
\hline $20-24$ & 1 & 1,17 \\
\hline $25-29$ & 3 & 3,52 \\
\hline $30-34$ & 2 & 2,35 \\
\hline $35-39$ & 2 & 2,35 \\
\hline $40-44$ & 3 & 3,52 \\
\hline $45-49$ & 10 & 11,76 \\
\hline $50-54$ & 9 & 10,58 \\
\hline $55-59$ & 8 & 9,41 \\
\hline $60-64$ & 8 & 9,41 \\
\hline $65-69$ & 9 & 10,58 \\
\hline $70-74$ & 13 & 15,29 \\
\hline $75-79$ & 6 & 7,05 \\
\hline $80-84$ & 6 & 7,05 \\
\hline $85-89$ & 2 & 2,35 \\
\hline $90-94$ & 2 & 2,35 \\
\hline
\end{tabular}

Los estudios diagnósticos para determinar la causa de disfagia fueron los siguientes: en 84 pacientes (99\%) se había realizado al menos EVDA (con biopsias) en algún momento; en otros casos se requirieron otros estudios complementarios a la EVDA, como pruebas funcionales (impedancia-pHmetría, manometría esofágica) o exámenes radiológicos (esofagografía o faringografía). Se observó entonces que 37 pacientes $(43,52 \%)$ únicamente requirieron EVDA, 47 (55,29\%) requirieron de endoscopia de vías digestivas altas en asociación con una prueba adicional y únicamente en un solo caso $(1 \%)$ no se requirió de EVDA para lograr determinar la causa de disfagia, y se llegó a esta por medio de pruebas radiológicas. También cabe mencionar que en 4 casos $(4,7 \%)$ se identificó la causa mediante EVDA en asociación con otras pruebas diferentes a las anteriormente mencionadas, como pruebas tomográficas, ultrasonográfica o paraclínicos serológicos específicos. En la Figura 2 se ilustran las pruebas que se requirieron a lo largo del tiempo para llegar a determinar la causa de disfagia.

Las causas de disfagia identificadas se muestran en la Tabla 2. Se encontró una mayor frecuencia de pacientes cuya causa de disfagia se asociaba con motilidad esofágica no efectiva; dentro de estas, las principales fueron la enfermedad por reflujo gastroesofágico (ERGE) $(30,58 \%)$ y el trastorno motor esofágico primario $(24,7 \%)$. En segundo

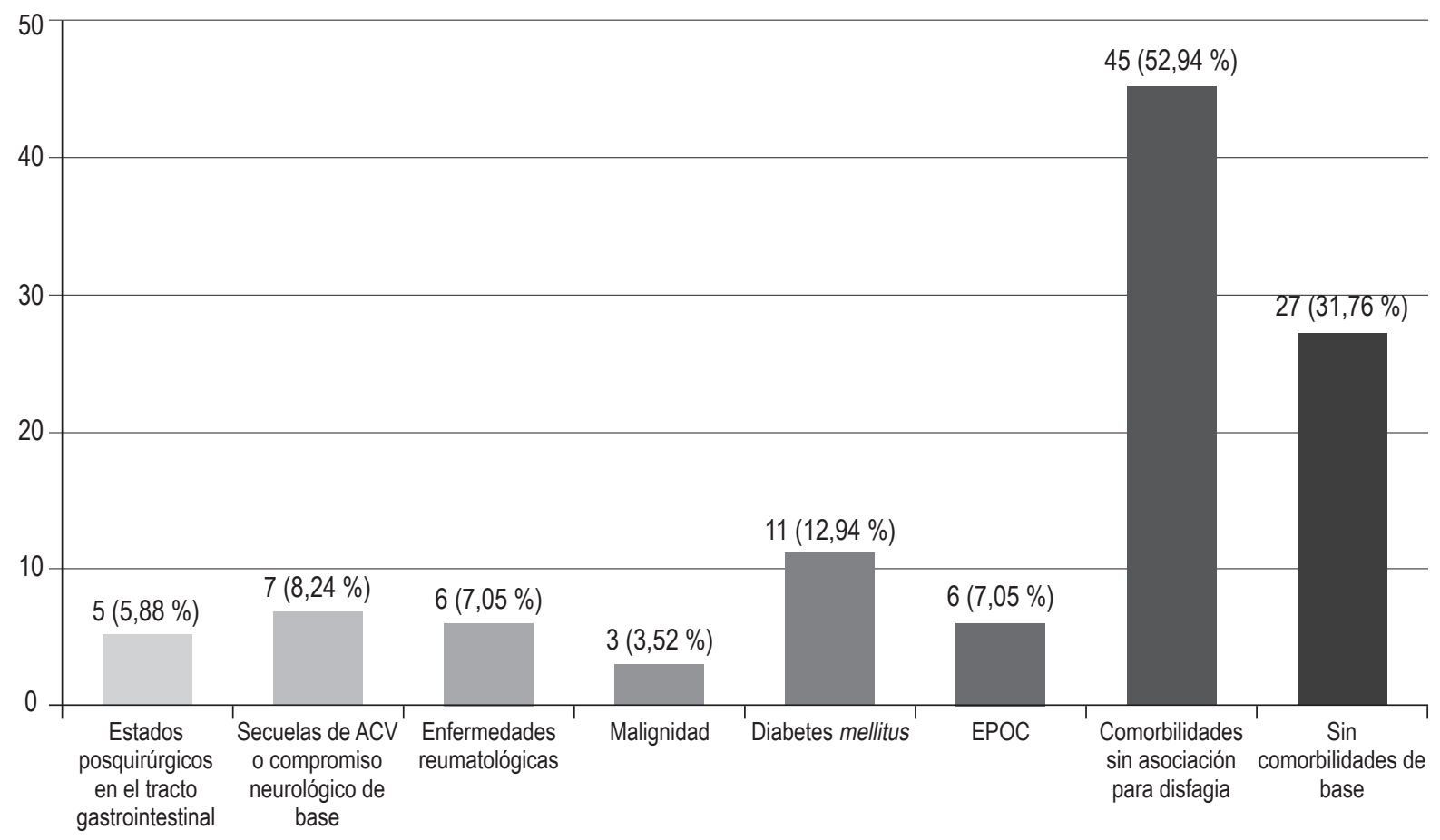

Figura 1. Principales comorbilidades encontradas en pacientes con disfagia $(n=85)$. ACV: accidente cerebrovascular; EPOC: enfermedad pulmonar obstructiva crónica. 


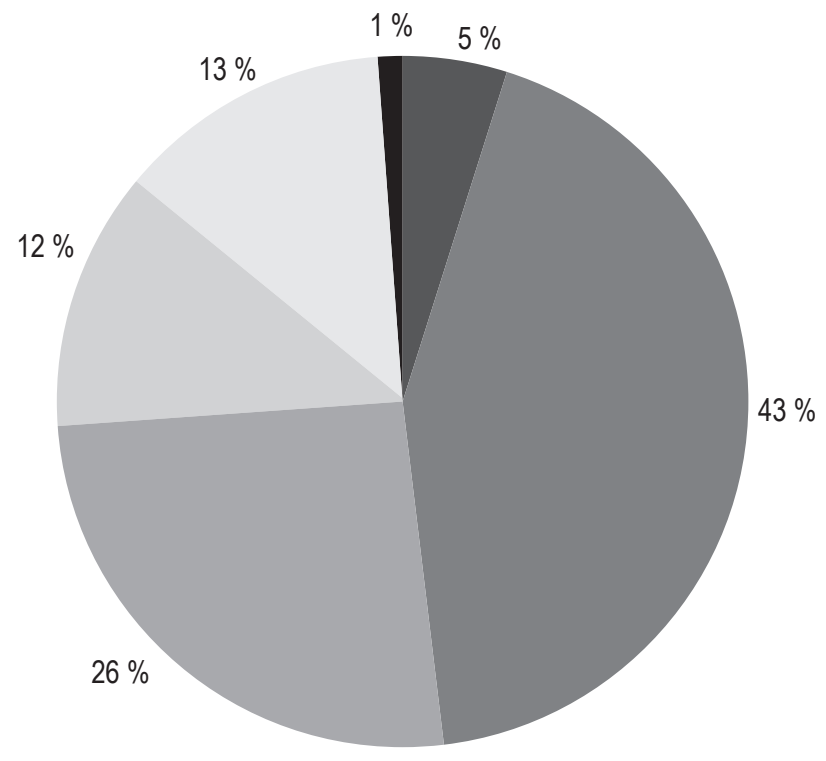

\section{Pruebas realizadas en los pacientes para identificar la causa de disfagia}

EVDA en asociación con otras pruebas (ultrasonografía, tomografía, serología, entre otras)

EVDA únicamente

EVDA en asociación con pruebas funcionales y radiográficas

EVDA en asociación con pruebas radiográficas

EVDA en asociación con pruebas funcionales

Faringografía por deglución

Figura 2. Pruebas realizadas en los pacientes para identificar la causa de disfagia $(\mathrm{n}=85)$.

lugar, con frecuencia se encontraron las anormalidades estructurales como tumores, estenosis péptica, anillos esofágicos o compresión extrínseca, que correspondieron al $28,23 \%$ de los casos (24 de 85 pacientes); dentro de esta categoría, los tumores ocuparon la mayor frecuencia encontrada, que correspondieron al 33,33 \% de esta misma ( 8 de 24 pacientes identificados). En menor proporción se encontró la asociación secundaria a causas inflamatorias, sea por esofagitis eosinofílica o esofagitis por radiación, que se identificó en 4 pacientes $(4,7 \%)$.

En la Tabla 3 se muestran las causas de disfagia de acuerdo con grupos de edad; la ERGE fue la principal causa de disfagia en la mayoría de grupos. Así mismo, se demuestra que el trastorno motor esofágico primario, la esofagitis eosinofílica y los tumores predominaron en grupos más jóvenes (menores de 60 años); mientras que las secuelas de desórdenes neurológicos, la estenosis péptica y el anillo de Schatzky predominaron en grupos de edad mayores.

Finalmente, se compararon las causas de disfagia de acuerdo con el sexo (Tabla 4) y se encontró que en mujeres hubo predominio de causas de disfagia relacionadas con desordenes autoinmunes e inflamación mucosa. Así mismo, en hombres especialmente, se encontraron causas inusuales de disfagia como disfagia lusoria, divertículo de Zenker y barra cricofaríngea.

\section{DISCUSIÓN}

Este trabajo constituye un aporte al conocimiento de la epidemiología de la disfagia en población adulta a nivel local.
Tabla 2. Causas identificadas de disfagia en pacientes que presentaron disfagia como motivo de consulta principal o síntoma asociado

\begin{tabular}{llcc}
\hline \multicolumn{1}{c}{$\begin{array}{c}\text { Tipo de } \\
\text { causa }\end{array}$} & \multicolumn{1}{c}{ Causa } & $\begin{array}{c}\text { Número de } \\
\text { pacientes }\end{array}$ & $\%$ \\
\hline Motilidad & & 56 & 67,03 \\
esofágica no & ERGE & 26 & 30,58 \\
efectiva & Trastorno motor esofágico & 21 & 24,7 \\
& primario & & \\
& Secuelas de desorden & 5 & 5,88 \\
& neurológico & & \\
& Secundaria a esclerodermia & 4 & 4,7 \\
& Secundaria a síndrome de & 1 & 1,17 \\
& Sjögren & & \\
Anormalidad & & 24 & 28,23 \\
& Tumores esofágicos & 8 & 9,41 \\
& Anillo de Schatzky & 7 & 8,23 \\
& Estenosis péptica & 6 & 7,05 \\
& Disfagia lusoria & 1 & 1,17 \\
& Divertículo de Zenker & 1 & 1,17 \\
& Barra cricofaríngea & 1 & 1,17 \\
& & 4 & 4,7 \\
& Esofagitis eosinofílica & 3 & 3,52 \\
& Postradioterapia & 1 & 1,17 \\
\hline Inflamación & & &
\end{tabular}

Indica que su prevalencia en pacientes no hospitalizados en un centro especializado en gastroenterología es del 2,7 \%. 
Tabla 3. Causas identificadas de disfagia de acuerdo con grupos de edad

\begin{tabular}{|c|c|c|c|c|c|c|c|c|}
\hline \multicolumn{9}{|c|}{ Edad (años) } \\
\hline $10-19(n=1)$ & $20-29(n=4)$ & $30-39(n=4)$ & $40-49(n=13)$ & $50-59(n=17)$ & $60-69(n=17)$ & $70-79(n=19)$ & $80-89(n=8)$ & $90-99(n=2)$ \\
\hline ERGE (1) & $\begin{array}{l}\text { Trastorno } \\
\text { motor } \\
\text { esofágico } \\
\text { primario (3) }\end{array}$ & ERGE (3) & ERGE (4) & ERGE (5) & ERGE (8) & $\begin{array}{l}\text { Trastorno } \\
\text { motor } \\
\text { esofágico } \\
\text { primario (7) }\end{array}$ & $\begin{array}{l}\text { Anillo de } \\
\text { Schatzky (2) }\end{array}$ & ERGE (1) \\
\hline \multirow[t]{15}{*}{$100 \%$} & $75 \%$ & $75 \%$ & $30,76 \%$ & $29,41 \%$ & $47,05 \%$ & $36,84 \%$ & $25 \%$ & $50 \%$ \\
\hline & ERGE (1) & $\begin{array}{l}\text { Trastorno } \\
\text { motor } \\
\text { esofágico } \\
\text { primario (1) }\end{array}$ & $\begin{array}{l}\text { Trastorno } \\
\text { motor } \\
\text { esofágico } \\
\text { primario (4) }\end{array}$ & $\begin{array}{l}\text { Tumores } \\
\text { esofágicos (5) }\end{array}$ & $\begin{array}{l}\text { Trastorno } \\
\text { motor } \\
\text { esofágico } \\
\text { primario (3) }\end{array}$ & $\begin{array}{l}\text { Secuelas de } \\
\text { desorden } \\
\text { neurológico } \\
\text { (3) }\end{array}$ & $\begin{array}{l}\text { Estenosis } \\
\text { péptica (2) }\end{array}$ & $\begin{array}{l}\text { Secuelas de } \\
\text { desorden } \\
\text { neurológico } \\
\text { (1) }\end{array}$ \\
\hline & $25 \%$ & $25 \%$ & $30,76 \%$ & $29,41 \%$ & $17,64 \%$ & $15,78 \%$ & $25 \%$ & $50 \%$ \\
\hline & & & $\begin{array}{l}\text { Secundaria a } \\
\text { esclerodermia } \\
(2)\end{array}$ & $\begin{array}{l}\text { Trastorno motor } \\
\text { esofágico } \\
\text { primario (2) }\end{array}$ & $\begin{array}{l}\text { Anillo de } \\
\text { Schatzky (2) }\end{array}$ & ERGE (3) & $\begin{array}{l}\text { Tumores } \\
\text { esofágicos } \\
\text { (2) }\end{array}$ & \\
\hline & & & $15,38 \%$ & $11,76 \%$ & $11,76 \%$ & $15,78 \%$ & $25 \%$ & \\
\hline & & & $\begin{array}{l}\text { Esofagitis } \\
\text { eosinofílica (2) }\end{array}$ & $\begin{array}{l}\text { Estenosis } \\
\text { péptica (1) }\end{array}$ & $\begin{array}{l}\text { Síndrome de } \\
\text { Sjögren (1) }\end{array}$ & $\begin{array}{l}\text { Estenosis } \\
\text { péptica (2) }\end{array}$ & $\begin{array}{l}\text { Secundaria } \\
\text { a desorden } \\
\text { neurológico } \\
\text { (1) }\end{array}$ & \\
\hline & & & $15,38 \%$ & $5,88 \%$ & $5,88 \%$ & $10,52 \%$ & $12,5 \%$ & \\
\hline & & & $\begin{array}{l}\text { Anillo de } \\
\text { Schatzky (1) }\end{array}$ & $\begin{array}{l}\text { Esofagitis } \\
\text { eosinofílica (1) }\end{array}$ & $\begin{array}{l}\text { Tumores } \\
\text { esofágicos (1) }\end{array}$ & $\begin{array}{l}\text { Disfagia } \\
\text { lusoria (1) }\end{array}$ & $\begin{array}{l}\text { Trastorno } \\
\text { motor } \\
\text { esofágico } \\
\text { primario (1) }\end{array}$ & \\
\hline & & & $7,6 \%$ & $5,88 \%$ & $5,88 \%$ & $5,26 \%$ & $12,5 \%$ & \\
\hline & & & & $\begin{array}{l}\text { Esclerodermia } \\
\text { (1) }\end{array}$ & $\begin{array}{l}\text { Esclerodermia } \\
\text { (1) }\end{array}$ & $\begin{array}{l}\text { Barra } \\
\text { cricofaríngea } \\
\text { (1) }\end{array}$ & & \\
\hline & & & & $5,88 \%$ & $5,88 \%$ & $5,26 \%$ & & \\
\hline & & & & $\begin{array}{l}\text { Anillo de } \\
\text { Schatzky (1) }\end{array}$ & $\begin{array}{l}\text { Estenosis } \\
\text { péptica (1) }\end{array}$ & $\begin{array}{l}\text { Anillo de } \\
\text { Schatzky (1) }\end{array}$ & & \\
\hline & & & & $5,88 \%$ & $5,88 \%$ & $5,26 \%$ & & \\
\hline & & & & $\begin{array}{l}\text { Postradioterapia } \\
\text { (1) }\end{array}$ & & $\begin{array}{l}\text { Divertículo de } \\
\text { Zenker (1) }\end{array}$ & & \\
\hline & & & & $5,88 \%$ & & $5,26 \%$ & & \\
\hline
\end{tabular}

A pesar de las limitaciones metodológicas, la información obtenida incluyó grupos de pacientes más jóvenes y es relevante para tener en cuenta en el ambiente extrahospitalario. Algunas fuentes han reportado que la disfagia puede alcanzar una prevalencia del $6 \%$ al $9 \%$ en la población general $(11,12)$. Sin embargo, se debe mencionar que la mayoría de estudios ha incluido pacientes mayores a 50 años. En el estudio de Eslick y colaboradores (7), se evaluaron a 672 sujetos con edad mayor de 18 años, y se encontró que el $16 \%$ había presentado alguna vez disfagia y un pico de presentación relacionado con la edad, ocurriendo de modo más frecuente entre los 40-49 años. En el presente estudio la prevalencia fue más baja y se encontró el pico de edad de presentación entre los 70-79 años. 
Tabla 4. Causas identificadas de disfagia de acuerdo con el sexo

\begin{tabular}{ll}
\hline \multicolumn{1}{c}{ Mujeres ( $\mathbf{n}=\mathbf{6 0}$ ) } & \multicolumn{1}{c}{ Hombres ( $\mathbf{~ = ~ 2 5 ) ~}$} \\
\hline ERGE (17) & ERGE (9) \\
$28,33 \%$ & $36 \%$ \\
Trastorno motor esofágico & Trastorno motor esofágico \\
primario (16) & primario (5) \\
$26,66 \%$ & $20 \%$ \\
Anillo de Schatzky (6) & Tumores esofágicos (4) \\
$10 \%$ & $16 \%$ \\
Secundaria a desorden & Estenosis péptica (2) \\
neurológico (5) & \\
$8,33 \%$ & $8 \%$ \\
Tumores esofágicos (4) & Esofagitis eosinofilica (1) \\
$6,66 \%$ & $4 \%$ \\
Estenosis péptica (4) & Anillo de Schatzky (1) \\
$6,66 \%$ & $4 \%$ \\
Esclerodermia (4) & Disfagia lusoria (1) \\
$6,66 \%$ & $4 \%$ \\
Esofagitis eosinofílica (2) & Divertículo de Zenker (1) \\
$3,33 \%$ & $4 \%$ \\
Postradioterapia (1) & Barra cricofaríngea (1) \\
$1,66 \%$ & $4 \%$ \\
Síndrome de Sjögren (1) & \\
$1,66 \%$ & \\
\hline
\end{tabular}

En la mayor parte de sujetos del estudio se identificó la ERGE como causa relacionada con disfagia, encontrándose en el 30,58 \% de los casos. La asociación de ERGE y disfagia se ha descrito previamente. En el estudio de Cho y colaboradores se evaluó la presencia de disfagia en 7640 pacientes, encontrando prevalencia de disfagia del $3 \%$, y la ERGE como principal diagnóstico asociado en un $30 \%$ de los casos (1). Otro estudio realizado por Hoy y colaboradores (13), en el que 100 pacientes con disfagia fueron valorados en un centro de atención ambulatorio especializado en trastornos de la deglución; durante un período de 15 meses se encontró que la edad media de presentación fue de 62 años y $27 \%$ de los pacientes presentaron ERGE. Bollschweiler y colaboradores (14) en un estudio comparativo demostraron que la disfagia aumentaba significativamente en el grupo con reflujo (28\%) frente al grupo control (3\%) y que esta asociación podría explicarse por peristalsis inadecuada inducida por ácido gástrico. Vakil y colaboradores (15) estudiaron la prevalencia de disfagia en 11945 pacientes con esofagitis erosiva y se encontró que el $37 \%$ de estos pacientes reportó disfagia.
Los tumores conformaron una parte significativa de las causas relacionadas con disfagia, por lo que los resultados son consistentes con la literatura de acuerdo con los diferentes grupos de edad (16). Así mismo, se encontró mayor prevalencia de disfagia en adultos mayores (grupos de edad mayores de 60 años), aunque se sabe que algunas condiciones médicas pueden predisponer a disfagia (desórdenes neurológicos) (17); en el presente estudio, que solo tuvo en cuenta población atendida en un centro gastroenterológico, se encontraron en menor proporción frente a ERGE y al trastorno motor esofágico primario.

Se destaca que gran parte de la población con disfagia correspondió a mujeres (70,5\% de los casos), aunque se sabe que las mujeres son más prestas a solicitar atención médica, a recibir recomendaciones para pruebas diagnósticas y a lograr adherencia a un tratamiento (18). A partir de algunos estudios se ha concluido que no existe alguna explicación biológica para esta diferencia de sexos y que las mujeres tienen mayor probabilidad para reportar síntomas gastrointestinales $(18,19)$. En el presente estudio se corrobora el hecho de que las mujeres son más susceptibles a reportar problemas de la deglución que los hombres, por lo que se requiere de mayor investigación para aclarar esta asociación.

Por último, cabe mencionar acerca de las pruebas requeridas para identificar la causa de disfagia. Se sabe que la historia médica y valoración física del paciente pueden dirigir a la prueba apropiada o remisión indicada (20). En el presente estudio, dado que se trató de un centro especializado en gastroenterología, sugiere que la mayoría de pacientes al momento de la remisión presentaba predominantemente síntomas sugestivos de obstrucción mecánica, por lo que la prueba de preferencia fue la endoscopia digestiva. En menor proporción se encontró la asociación de otros métodos diagnósticos como las pruebas funcionales y radiográficas, lo cual está en relación con que la mayoría de pacientes identificados presentó diagnósticos de trastornos en la motilidad esofágica, lo que representa entonces una limitación y sugiere que para futuros estudios al momento de valorar las características clínicas de la disfagia debe incluirse información de otras especialidades como otorrinolaringología, fonoaudiología, cirugía de cabeza y cuello, y neurología, entre otras.

\section{CONCLUSIONES}

La prevalencia de disfagia en el contexto de atención ambulatoria en la institución es similar a la reportada en la literatura. Los trastornos por motilidad esofágica no efectiva correspondieron a los diagnósticos más frecuentes, corroborándose la asociación de ERGE con disfagia. Al incluirse grupos más jóvenes, la disfagia continúa siendo un problema pre- 
dominantemente de adultos mayores ( $>65$ años). A su vez, representa un problema cuyo enfoque es multidisciplinario, que incluye otras especialidades diferentes a gastroenterología. Y se requieren de mayores esfuerzos para caracterizar la epidemiología de la disfagia a nivel nacional.

\section{Conflicto de intereses}

Los autores no declaran ningún conflicto de intereses.

\section{Financiación}

Los autores no recibieron ningún tipo de financiación para la realización de este estudio.

\section{Agradecimientos}

Ninguno declarado por los autores.

\section{REFERENCIAS}

1. Cho SY, Choung RS, Saito YA, Schleck CD, Zinsmeister $A R$, Locke GR 3rd, et al. Prevalence and risk factors for dysphagia: a USA community study. Neurogastroenterol Motil. 2015;27(2):212-9. doi: 10.1111/nmo.12467.

2. Achem SR, Devault KR. Dysphagia in aging. J Clin Gastroenterol. 2005;39(5):357-71.

3. Lawal A, Shaker R. Esophageal dysphagia. Phys Med Rehabil Clin N Am. 2008;19(4):729-45, viii. doi: 10.1016/j. pmr.2008.07.003.

4. Mann G, Hankey GJ, Cameron D. Swallowing function after stroke: prognosis and prognostic factors at 6 months. Stroke. 1999;30(4):744-8.

5. Lindgren S, Janzon L. Prevalence of swallowing complaints and clinical findings among 50-79-year-old men and women in an urban population. Dysphagia. 1991;6(4):187-92.

6. Drossman DA, Li Z, Andruzzi E, Temple RD, Talley NJ, Thompson WG, et al. U.S. householder survey of functional gastrointestinal disorders. Prevalence, sociodemography, and health impact. Dig Dis Sci. 1993;38(9):1569-80.

7. Eslick GD, Talley NJ. Dysphagia: epidemiology, risk factors and impact on quality of life--a population-based study. Aliment Pharmacol Ther. 2008;27(10):971-9. doi: 10.1111/j.1365-2036.2008.03664.x.
8. Shanley C, O'Loughlin G. Dysphagia among nursing home residents: an assessment and management protocol. J Gerontol Nurs. 2000;26(8):35-48.

9. Park YH, Han HR, Oh BM, Lee J, Park JA, Yu SJ, et al. Prevalence and associated factors of dysphagia in nursing home residents. Geriatr Nurs. 2013;34(3):212-7. doi: 10.1016/j.gerinurse.2013.02.014.

10. Tsukamoto M, Manabe N, Kamada T, Hirai T, Hata J, Haruma K, et al. Number of Gastrointestinal Symptoms is a Useful Means of Identifying Patients with Cancer for Dysphagia. Dysphagia. 2016;31(4):547-54. doi: 10.1007/ s00455-016-9712-z.

11. Lind CD. Dysphagia: evaluation and treatment. Gastroenterol Clin North Am. 2003;32(2):553-75.

12. Brett A. Screening test for stroke-related dysphagia. J Watch Gen Med. 2009;29:166-7.

13. Hoy M, Domer A, Plowman EK, Loch R, Belafsky P. Causes of dysphagia in a tertiary-care swallowing center. Ann Otol Rhinol Laryngol. 2013;122(5):335-8. doi: 10.1177/000348941312200508.

14. Bollschweiler E, Knoppe K, Wolfgarten E, Hölscher AH. Prevalence of dysphagia in patients with gastroesophageal reflux in Germany. Dysphagia. 2008;23(2):172-6. doi: 10.1007/s00455-007-9120-5.

15. Vakil NB, Traxler B, Levine D. Dysphagia in patients with erosive esophagitis: prevalence, severity, and response to proton pump inhibitor treatment. Clin Gastroenterol Hepatol. 2004;2(8):665-8.

16. Roden DF, Altman KW. Causes of dysphagia among different age groups: a systematic review of the literature. Otolaryngol Clin North Am. 2013;46(6):965-87. doi: 10.1016/j.otc.2013.08.008.

17. Marik PE, Kaplan D. Aspiration pneumonia and dysphagia in the elderly. Chest. 2003;124(1):328-36.

18. Wilkins T, Gillies RA, Thomas AM, Wagner PJ. The prevalence of dysphagia in primary care patients: a HamesNet Research Network study. J Am Board Fam Med. 2007;20(2):144-50. doi: 10.3122/jabfm.2007.02.060045.

19. Heitkemper M, Jarrett M, Bond EF, Chang L. Impact of sex and gender on irritable bowel syndrome. Biol Res Nurs. 2003;5(1):56-65. doi: 10.1177/1099800403005001006.

20. Spieker MR. Evaluating dysphagia. Am Fam Physician. 2000;61(12):3639-48. 